\title{
An intrinsic biochemical concentration sensor using a polymer optical fibre Bragg grating
}

\author{
W. Zhang ${ }^{* a}$, D. J. Webb ${ }^{\text {a }}$ G. -D. Peng ${ }^{\mathrm{b}}$ \\ ${ }^{a}$ Aston Institute of Photonic Technologies, Aston University, Birmingham, B4 7ET, UK \\ ${ }^{\mathrm{b}}$ School of Electrical Engineering and Telecommunications, University of New South Wales, \\ Sydney, NSW 2052, Australia
}

\begin{abstract}
A new type of fibre-optic biochemical concentration sensor based on a polymer optical fibre Bragg grating (POFBG) is proposed. The wavelength of the POFBG varies as a function of analyte concentration. The feasibility of this sensing concept is demonstrated by a saline concentration sensor. When polymer fibre is placed in a water based solution the process of osmosis takes place in this water-fibre system. An osmotic pressure which is proportional to the solution concentration, will apply to the fibre in addition to the hydraulic pressure. It tends to drive the water content out of the fibre and into the surrounding solution. When the surrounding solution concentration increases the osmotic pressure increases to drive the water content out of the fibre, consequently increasing the differential hydraulic pressure and reducing the POFBG wavelength. This process will stop once there is a balance between the osmotic pressure and the differential hydraulic pressure. Similarly when the solution concentration decreases the osmotic pressure decreases, leading to a dominant differential hydraulic pressure which drives the water into the fibre till a new pressure balance is established. Therefore the water content in the polymer fibre - and consequently the POFBG wavelength - depends directly on the solution concentration. A POFBG wavelength change of $0.9 \mathrm{~nm}$ was measured for saline concentration varying from 0 to $22 \%$. For a wavelength interrogation system with a resolution of $1 \mathrm{pm}$, a measurement of solution concentration of $0.03 \%$ can be expected.
\end{abstract}

Key words: Fibre Bragg grating, PMMA, polymer swelling, solution concentration, osmosis process

\section{INTRODUCTION}

Optical fibre has become an important part of sensor technology. Low loss light delivery, long interaction length, and compatibility with time/wavelength multiplexing allow it to take advantage of technology developed for optical communications. Fibre Bragg grating (FBG) sensors inscribed in silica optical fibre have become an increasingly mature sensing technology. FBGs have also been used for biochemical sensing, where the usual aim is to produce a signal that is ideally proportional to the concentration of a chemical or biochemical species. However silica is itself quite inert and it is consequently necessary to add additional fibre coatings to provide the necessary biochemical sensitivity [1]. More recently, FBG sensors have been inscribed into poly(methyl methacrylate) (PMMA) based plastic optical fibre in both step-index [2] and microstructured geometries [3]. The physical and chemical properties of polymeric materials are rather different to silica and may offer advantages in certain situations. An interesting feature of PMMA is the affinity for water which leads to a swelling of the fibre and an increase of index, both of which contribute to an increase in the Bragg wavelength of a FBG written in the fibre [4].

In this work a new type of fibre-optic biochemical concentration sensor is proposed. The sensing element is a Bragg grating inscribed in polymer optical fibre which swells or shrinks as a function of analyte concentration. Swelling or shrinking changes the wavelength of polymer optical fibre grating. Changes in analyte concentration are detected as changes in Bragg wavelength. The feasibility of this sensing concept is demonstrated by a saline concentration sensor.

*w.zhang@aston.ac.uk

Microstructured and Specialty Optical Fibres, edited by Kyriacos Kalli, Alexis Mendez,

Proc. of SPIE Vol. 8426, 842618 - (c) 2012 SPIE · CCC code: 0277-786X/12/\$18 · doi: 10.1117/12.922279

Proc. of SPIE Vol. 8426 842618-1 


\section{EXPERIMENTS}

The experimental setup is shown in Fig. 1. The POFBG was fabricated by illuminating from above a phase mask placed on top of PMMA based POF using $325 \mathrm{~nm}$ light from a HeCd laser, which produced a Bragg wavelength in the $1550 \mathrm{~nm}$ region [3]. The grating was then attached to a singlemode silica fibre down-lead using UV curable glue. In the experiment the POFBG was inserted into test tubes of saline solution with different concentrations. The reflection of the POF grating was monitored by using an IBSEN I-MON 400 wavelength interrogation system. In the experiment the POFBG was placed in an environmental chamber with the humidity set at $\sim 45 \%$ and the temperature at $21{ }^{\circ} \mathrm{C}$ and its wavelength monitored by the wavelength interrogation system.

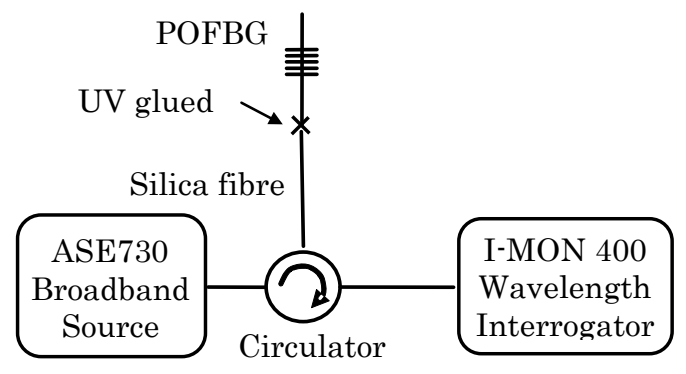

Fig. 1 Experimental arrangement

The POFBG was first inserted into a test tube of distilled water for around 2 hours, so the POFBG was fully swelled by the water, then pulled out and inserted into a test tube of saline solution. Four samples of saline solution $(7.5 \%, 13 \%$, $17.5 \%$ and $22 \%$ ) were tested in the order of ascending concentrations, then in the order of descending concentration. The POFBG device was left for 1 hour in each sample to allow equilibrium to be reached. The POFBG wavelength was monitored with an interval of 60 seconds. The POFBG wavelength over the complete experimental process was recorded as shown in Fig. 2.

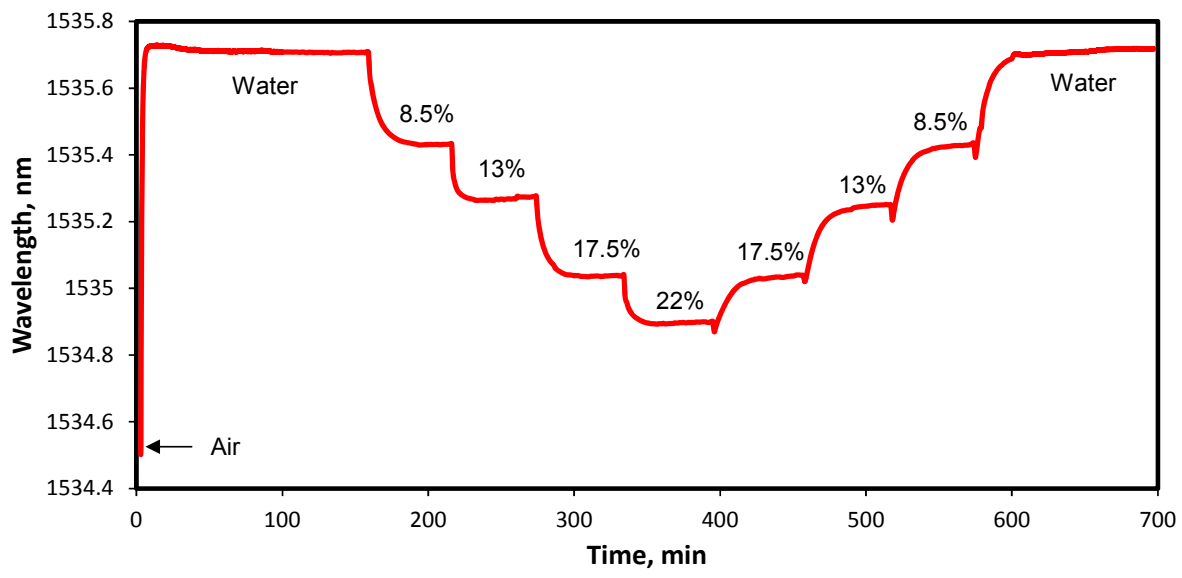

Fig. 2 Wavelength change of POFBG over complete experimental process

From the captured response one can see that the POFBG wavelength rose rapidly when the grating was inserted into the water; it took some time for the grating to stabilize its wavelength in water. Then the POFBG wavelength varied significantly as a function of saline concentration. The POFBG wavelength decreased with increased saline concentration, and then increased with the decreased salt concentration. The process is reversible. 


\section{DISCUSSIONS}

It is well known that PMMA and many other polymers have the tendency to absorb water $[4,5]$. Many polymers show water absorption processes, where clustering of water occurs within the polymer matrix leading to an absorption increase at higher humidities. This process leads to a swelling of the fibre and an increase of index, both of which contribute to an increase in the Bragg wavelength of a FBG written in the fibre. The water content absorbed in fibre closely depends on the relative humidity surrounding the fibre. This is the operation principle for the POFBG humidity sensor [6].

The wavelength of the polymer fibre grating can be related to the water content in the polymer fibre. It is assumed that the Bragg wavelength shifts linearly with water content in the polymer fibre, $W[4,7]$. Therefore the Bragg wavelength can then be expressed as

$$
\lambda_{B}=\lambda_{B o}+\alpha W
$$

where $\lambda_{B 0}$ is the initial Bragg wavelength and $\alpha$, a coefficient associated with PMMA swelling, water dependence of the refractive index, and swelling induced stress. The process of water absorption or desorption in PMMA can be described by the diffusion theory of mass transfer [8].

When the polymer fibre is inserted in water the fibre is fully swollen, giving rise to a maximum wavelength change of the POFBG. If solute exists in the water then the water content absorbed in the polymer fibre will change, due to the process of osmosis [9]. In this case the polymer fibre acts as a semipermeable membrane, which allows solvent (water in this case) to pass through and blocks the solute (salt in this case). The osmotic pressure generated by a solution with a molar concentration, $c$, is simply given by [10]

$$
P_{\text {osm }}=c R T
$$

where $R$ is the gas constant $\left(8.314 \mathrm{~J} \cdot \mathrm{K}^{-1} \cdot \mathrm{mol}^{-1}\right)$ and $T$ the temperature. The rate of water transportation between fibre and surrounding solution can be expressed as

$$
Q=\left(\Delta P-P_{o s m}\right) K_{w} S / d
$$

where $\Delta P$ is a differential hydraulic pressure, generated by the water pressure difference inside and outside the fibre, $K_{w}$, the membrane permeability coefficient for water, $S$, the membrane area, and $d$, the membrane thickness. If outside water pressure is higher than inside water pressure $\Delta P$ is positive, driving the water into the polymer fibre. The differential hydraulic pressure will reduce to minimum and the fibre will be fully swollen in pure water. When the surrounding solution concentration increases, the osmotic pressure increases correspondingly to drive the water content in the fibre into the surrounding solution, consequently increasing the differential hydraulic pressure. This process will stop once there is a balance between the osmotic pressure and the differential hydraulic pressure. Similarly when the solution concentration decreases the osmotic pressure decreases, leading to a dominant differential hydraulic pressure which drives the water into the fibre till a new pressure balance is established. Therefore the water content in the polymer fibre depends directly on the solution concentration, which is readily monitored by the wavelength change of the POFBG.

The wavelength change against concentration is plotted in Fig. 3. From it one can see that the POFBG wavelength varies in a slightly non-linear fashion with the concentration. Similar nonlinearity has been noticed in [11] where the POFBG wavelength varies nonlinearly with relative humidity. 


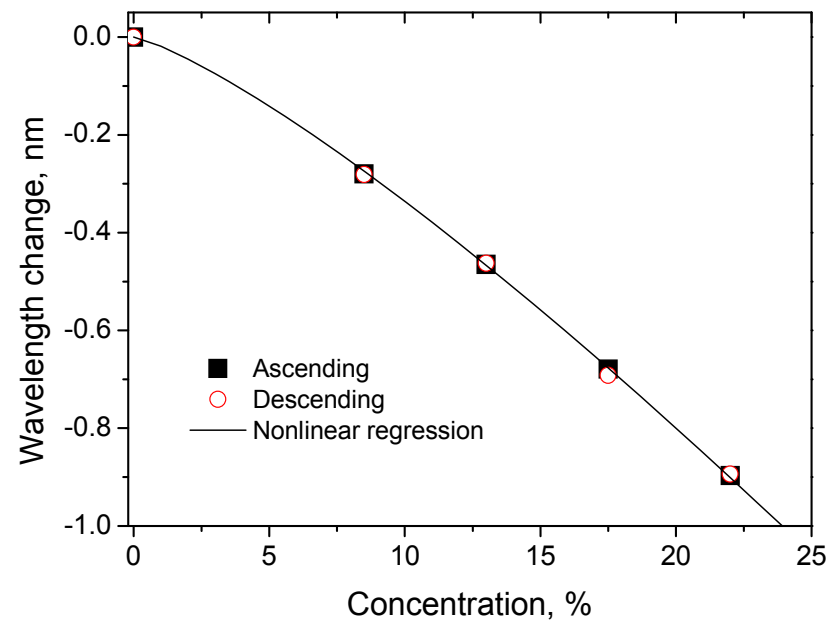

Fig. 3 Wavelength change of POFBG vs. concentration.

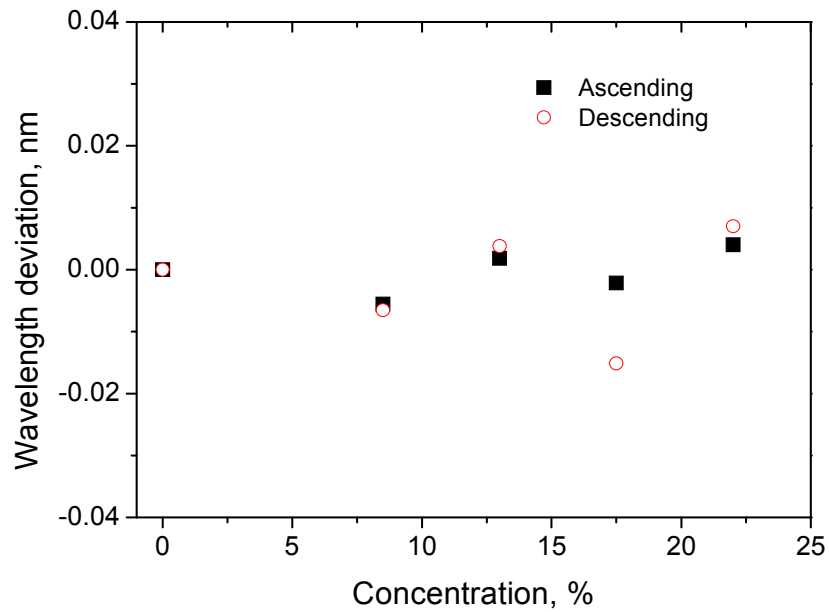

Fig. 4 Wavelength deviation for concentration measurement

This is attributed to the permeability coefficient for water of many polymeric materials that varies with relative humidity due to absorbed water induced plasticizing effect on polymer materials [12]. When the POFBG is placed in solution, osmotic pressure is generated, driving the water out of the fibre and thus a POFBG wavelength decrease take place. Meanwhile $\triangle P$ starts to increase to balance the osmotic pressure. So the POFBG wavelength exhibits a sharp drop first then the drop slows down. When $\Delta P$ equals the osmotic pressure the water exchange stops and the POFBG wavelength becomes stable. The osmotic pressure increases as the solution concentration increases, giving rise to an increased hydraulic pressure, thus a larger permeability coefficient for water. From (3) one can deduce that a larger permeability coefficient produces a higher rate of water transportation between fibre and surrounding solution. It leads to a nonlinear change of the water content absorbed in the fibre and thus a nonlinear Bragg wavelength change against relative water pressure. Non-linear regression was used to fit the response to an exponential function, the result being shown in Fig.3 where POFBG wavelength change against water concentration can be defined by $\Delta \lambda \propto-c^{m}$ with $m=1.25$. 
Fig. 4 shows the wavelength deviation from this regression for each concentration measurement. It can be noticed that in the measurements of both increased concentration and decreased concentration the POFBG wavelength shows almost the same value for the same sample. This performance shows that the sensor is reversible. Actually the plasticizing effects of water in polymers are completely reversible when the structure of the polymer remains unchanged and chemical transformations are insignificant [12]. When the saline concentration varies from 0 to $22 \%$ the corresponding wavelength shift of the POFBG sensor is $\sim 0.9 \mathrm{~nm}$. Considering the wavelength resolution of I-MON 400 wavelength interrogation system (better than $1 \mathrm{pm}$ ) a very accurate measurement of solution concentration can be achieved. A more detailed time response was captured with an interval of 10 seconds, as shown in Fig. 5. It can be seen that there is a large spike in the sensor response during each sample change. This is because when the sensor was pulled out of the solution and exposed to air the POFBG wavelength dropped sharply due to large negative differential hydraulic pressure between fibre and air, driving the water out of the fibre.

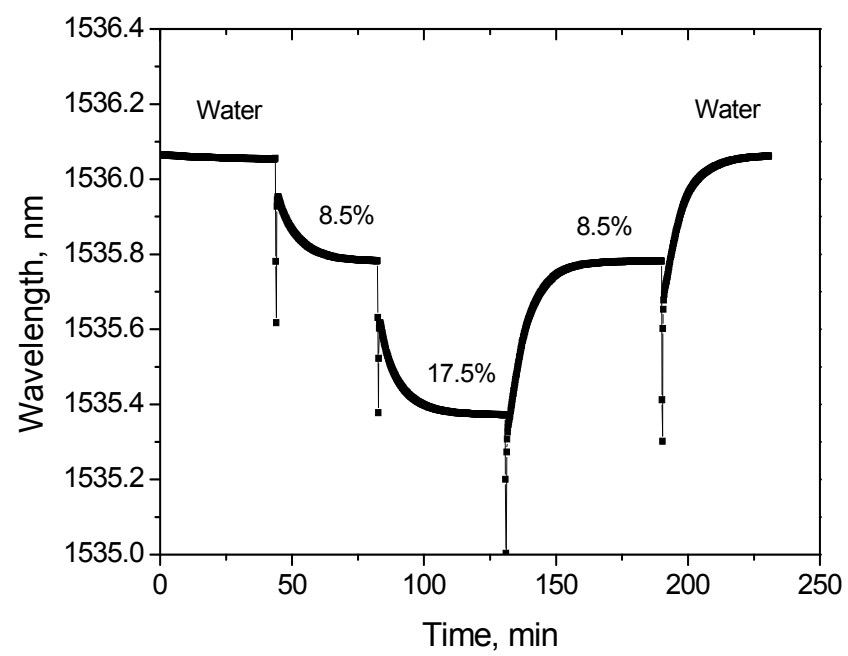

Fig. 5 A more detailed sensor response against step change of saline concentration

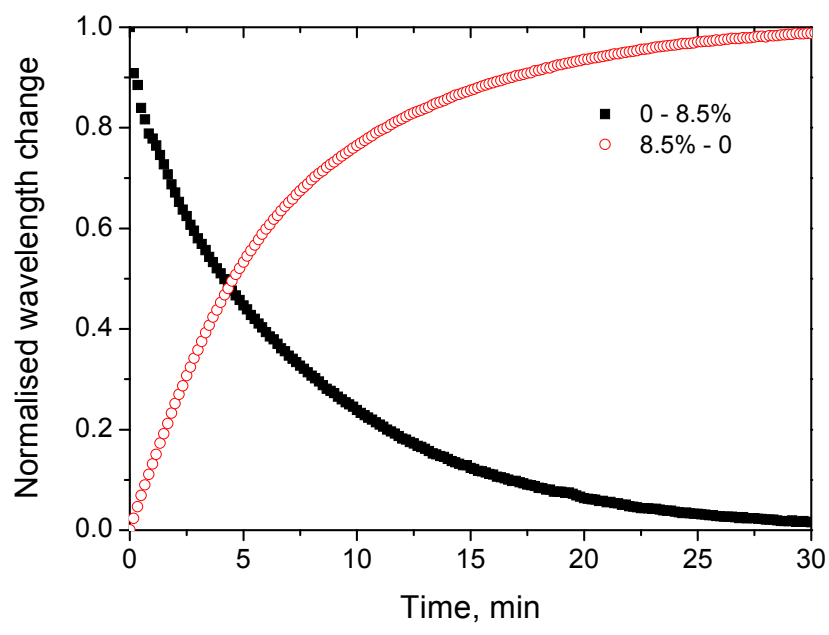

Fig. 6 Sensor responses reflecting response time 
It takes some time for the sensor response to reach a stable value. The sensor response to a step concentration change can be approximated by an exponential function and a response time can be estimated as the time being taken to reach $90 \%$ of the equilibrium value. Fig. 6 shows the relative wavelength changes for the saline concentration changes of 0 to $8.5 \%$ and $8.5 \%$ to 0 . The estimated response times are $17.3 \mathrm{~min}$ and $16 \mathrm{~min}$, respectively. It should be pointed out that according to the diffusion theory of mass transfer [8] the response time strongly depends on the diameter and polymer material of the fibre used. For the same type of polymer fibre, it has been demonstrated that a smaller diameter leads to a faster response time [11]. The fibre diameter used in this work is $135 \mu \mathrm{m}$, which was achieved by using acetone etching [11]. Further reducing fibre diameter could destabilize the grating operation. A much improved response time can be expected by using a grating inscribed in slotted PMMA optical fibre [14] in which water could reach the fibre core in much less time.

\section{CONCLUSION}

A polymer fibre Bragg grating based biochemical concentration sensor has been proposed and demonstrated in the measurement of saline concentration. Unlike most fibre optic biosensors this sensor does not require an immobilized indicator dye therefore the performance is not limited by the stability and optical properties of that indicator [2]. The sensor is reversible and thus can be used for in-situ repeatable measurement and monitoring of concentration. The grating can be inscribed to provide a response in the near infrared region to take full advantage of technology developed for fibre-optic communications. The operation principle means that this concept can be used for sensing other singlesolute biochemical solutions. An interesting feature is that the sensing concept is based on the process of osmosis, which is a natural process in many living cells. From this point of view the device is a real biosensor.

\section{REFERENCES}

[1] Bosch, M. E., Sánchez, A. J. R., Rojas, F. S., and Ojeda, C. B., "Recent Development in Optical Fibre Biosensors," Sensors, 7, 797-859 (2007)

[2] Xiong, Z., Peng, G. D., Wu, B., and Chu, P. L., "Highly tunable Bragg gratings in single-mode polymer optical fibres," IEEE Photon. Tech. Lett., 11(3), 352-354 (1999)

[3] Dobb, H., Webb, D. J., Kalli, K., Argyros, A., J. Large, M. C., and van Eijkelenborg, M. A. ,” Continuous wave ultraviolet light-induced fibre Bragg gratings in few- and single-mode microstructured polymer optical fibres," Opt. Lett., 30, 3296$3298(2005)$

[4] Harbach, N. G., "Fibre bragg gratings in polymer optical fibres." PhD thesis Lausanne: EPFL (2008).

[5] Barrie, J. A. and Platt, B., "The Diffusion and Clustering of Water Vapour in Polymers," Polymer, 4, p.303 (1963).

[6] Zhang, C., Zhang, W., Webb, D. J., Peng, G.-D., "Optical fibre temperature and humidity sensor", Electron. Lett. 46, pp. 643-644 (2010)

[7] Thomas, A. M., "Moisture Permeability, Diffusion and Sorption in Organic Film-Forming Materials," Journal of Applied Chemistry, 1, 141-146 (1951).

[8] Crank, J., [The Mathematics of Diffusion], 2nd ed., Clarendon Press - Oxford (1975).

[9] van 't Hoff, J. H., "Osmotic pressure and chemical equilibrium," Nobel Prize lecture (1901)

[10] Hiemenz, P. C., and Lodge, T. P., [Polymer Chemistry: The Basic Concepts], $2^{\text {nd }}$ ed. CRC Press (2007).

[11] Zhang, W., Webb, D. J., Peng, G.-D., "Investigation into Time Response of Polymer Fibre Bragg Grating Based Humidity Sensors," IEEE J. Lightwave Tech 30(8), 1090 - 1096 (2012)

[12] Comyn, J., [Polymer Permeability], Elsevier Applied Science Publishers, London (1985).

[13] Cox, F. M., Large, M. C. J., Cordeiro, C. M. B., Lwin, R., Argyros, A., "Slotted microstructured optical fibre," Proc. of SPIE, 7004 700427-1 (2007) 